\title{
Preoperative chemoradiotherapy versus postoperative chemoradiotherapy for stage II-III resectable rectal cancer: a meta-analysis of randomized controlled trials
}

\author{
Jin Ho Song, MD', Jae Uk Jeong, MD², Jong Hoon Lee, MD³, Sung Hwan Kim, MD³, \\ Hyeon Min Cho, MD', Jun Won Um, MD ${ }^{5}$, Hong Seok Jang, MD ${ }^{6}$ for Korean Clinical Practice Guideline for \\ Colon and Rectal Cancer Committee
}

\begin{abstract}
'Department of Radiation Oncology, Gyeongsang National University Hospital, Gyeongsang National University School of Medicine, Jinju; ${ }^{2}$ Department of Radiation Oncology, Chonnam National University Hospital, Chonnam National University School of Medicine, Gwangju; Departments of ${ }^{3}$ Radiation Oncology and ${ }^{4}$ Surgery, St. Vincent's Hospital, College of Medicine,

The Catholic University of Korea, Suwon; ${ }^{5}$ Department of Surgery, Korea University Ansan Hospital, Ansan;

${ }^{6}$ Department of Radiation Oncology, Seoul St. Mary's Hospital, College of Medicine, The Catholic University of Korea, Seoul, Korea
\end{abstract}

Purpose: Whether preoperative chemoradiotherapy (CRT) is better than postoperative CRT in oncologic outcome and toxicity is contentious in prospective randomized clinical trials. We systematically analyze and compare the treatment result, toxicity, and sphincter preservation rate between preoperative CRT and postoperative CRT in stage II-III rectal cancer.

Materials and Methods: We searched Medline, Embase, and Cochrane Library from 1990 to 2014 for relevant trials. Only phase III randomized studies performing CRT and curative surgery were selected and the data were extracted. Meta-analysis was used to pool oncologic outcome and toxicity data across studies.

Results: Three randomized phase III trials were finally identified. The meta-analysis results showed significantly lower 5-year locoregional recurrence rate in the preoperative-CRT group than in the postoperative-CRT group (hazard ratio, 0.59; 95\% confidence interval, $0.41-0.84 ; p=0.004)$. The 5 -year distant recurrence rate $(p=0.55)$, relapse-free survival $(p=0.14)$, and overall survival $(p=0.22)$ showed no significant difference between two groups. Acute toxicity was significantly lower in the preoperativeCRT group than in the postoperative-CRT group $(p<0.001)$. However, there was no significant difference between two groups in perioperative and chronic complications $(p=0.53)$. The sphincter-saving rate was not significantly different between two groups ( $p$ $=0.24$ ). The conversion rate from abdominoperineal resection to low anterior resection in low rectal cancer was significantly higher in the preoperative-CRT group than in the postoperative-CRT group $(p<0.001)$.

Conclusions: As compared to postoperative CRT, preoperative CRT improves only locoregional control, not distant control and survival, with similar chronic toxicity and sphincter preservation rate in rectal cancer patients.

Keywords: Chemoradiotherapy, Preoperative, Postoperative, Rectal cancer, Surgery

Received 01 February 2017, Revised 29 March 2017, Accepted 31 March 2017.

Correspondence: Jong Hoon Lee, MD, Department of Radiation Oncology, St. Vincent's Hospital, College of Medicine, The Catholic University of Korea, 93 Jungbu-daero, Paldal-gu, Suwon 16247, Korea. Tel: +82-31-249-8440, Fax: +82-31-257-3734, E-mail: koppul@catholic.ac.kr

(c) This is an Open Access article distributed under the terms of the Creative Commons Attribution Non-Commercial License (http://creativecommons.org/ licenses/by-nc/4.0/) which permits unrestricted non-commercial use, distribution, and reproduction in any medium, provided the original work is properly cited.

www.e-roj.org 


\section{Introduction}

Colorectal cancer is the fourth most common form of malignancy worldwide, with an estimated 800,000 new cases being diagnosed each year. Colorectal cancer accounts for about 10\% of all cancers [1]. Although the incidence of colorectal cancer has been decreased since 2000 in the United States, the incidence of colorectal cancer is still increasing in Asian countries.

Since surgical view and access to the pelvic cavity is restricted and lymphatic drainage is systemic and portal in rectal cancer, the treatment strategy and outcome of rectal cancer differs with that of colon cancer which surgical access is easy and lymphatic drainage is mostly portal [2]. Locoregional recurrence rate in rectal cancer is high after surgery alone. Therefore, several adjuvant therapies including radiation and chemotherapy have been investigated $[3,4]$. Due to these adjuvant treatments, the 5 -year relative survival rates have increased from $48.1 \%$ in the mid-1970s to $67.7 \%$ in the 2000s [1]. This survival improvement in rectal cancer was greatest for the stage II-III locally advanced disease according the introduction of concurrent chemoradiotherapy (CRT).

There are two approaches for the CRT administration in rectal cancer treatment. One is preoperative CRT, which is delivered before radical surgery when the tumor is 'clinically' T3-4 or node-positive. The other is postoperative CRT, which is administered after radical surgery when the tumor is 'pathologically' T3-4 or node-positive. Preoperative or postoperative CRT in rectal cancer have been proven to reduce local and systemic recurrences than radiation or chemotherapy alone in randomized controlled trials [3,4], and more favorable results in locoregional control and sphincter preservation for preoperative CRT than for postoperative CRT had been reported in the German trial $[5,6]$. Thus, the National Cancer Comprehensive Network (NCCN) guidelines have adopted preoperative CRT as the standard of care in stage II-III rectal cancer. However, whether preoperative CRT is better than postoperative CRT in oncologic outcome is contentious, since other phase III randomized trials did not have same results as those of the German trial [7-10]. Thus, in this study, we performed a meta-analysis of prospective phase III randomized trials in rectal cancer to compare the oncologic outcome, toxicity, and sphincter preservation rate between preoperative CRT and postoperative CRT.

\section{Materials and Methods}

\section{Literature search and selection criteria}

We searched for published and unpublished phase III randomized controlled trials comparing preoperative and postoperative CRT for patients with stage $\mid I-I I$ resectable rectal cancer. Patients aged 18 years and older were eligible for inclusion. All available chemotherapy regimens with radiotherapy were accepted. Only studies which curative surgery was performed were included. If local excision was performed as surgery, the study was excluded.

We searched Medline, Embase, and Cochrane Library from January 1990 to December 2014 for relevant trials. We also searched abstracts from the major European or American international oncologic meetings: ASTRO, ASCO, ESTRO, and ESMO. Electronic database searches were performed with MeSH terms (rectal neoplasms, colorectal neoplasms, chemoradiotherapy, and radiotherapy) and free text terms (rectal cancer, adenocarcinoma, neoplasm, radiotherapy, chemotherapy, chemoradiation, radiochemotherapy, preoperative, postoperative, and neoadjuvant). We restricted our searches to articles published in English. Two independent reviewers (Lee JH and Jeong JU) screened the title and abstract of searched articles. Trials that seemed to meet the inclusion and exclusion criteria were selected for full-text review.

\section{Outcome measures}

The following outcomes were evaluated: 5 -year overall survival (OS) and relapse-free survival (RFS), 5-year locoregional and distant recurrence rate, pathologic complete response (pCR), sphincter preservation, conversion rate from abdominoperineal resection (APR) to low anterior resection (LAR) in low-lying tumor, and acute and chronic toxicity.

OS was defined as time from curative surgery to death from any cause or to last follow-up (censored). RFS was defined as time to any recurrence or death or to last followup (censored) from curative surgery. pCR was defined as the complete absence of a viable tumor with only fibrotic mass in the pathologic specimen after CRT and surgery (ypTONO).

\section{Statistical analyses}

Two reviewers (Lee JH and Jeong JU) obtained the full text of relevant randomized controlled studies and assessed methodological quality according to the Cochrane Collaboration's tool for assessing the risk of bias. Methodological details relevant for potential bias included random sequence generation, allocation concealment, blinding of participants, personnel and outcome assessors, 
incomplete outcome data, selective outcome reporting, and other sources of bias. Disagreements between the two reviewers were resolved by discussion and consensus. Data were extracted by one reviewer (Lee JH) on custom-designed forms and entered in a computer database for transfer and statistical analysis in the Review Manager software (Lee JH and Song JH). The data extracted included first author, year of publication, source, sequence of CRT and surgery, clinical stage, number of patients included, and outcome parameters as listed above. Data accuracy was verified by the senior authors (Jang HS, Kim SH, Um JW, and Cho HM). Differences between categorical outcome parameters were quantified using the hazard ratio (HR) and corresponding 95\% confidence interval (Cl). Chi-square test and I-square test were used for testing heterogeneity between studies. If heterogeneity was not present ( $p>0.10$ and $12<50 \%$ ), fixed-effect model was adopted for data analysis. Otherwise, random-effect model will be employed. All statistical analyses were performed using RevMan Review Manager (ver. 5.3) and R (ver. 3.1.0). A p-value $<0.05$ was considered as statistically significant one.

\section{Results}

\section{Description of studies}

The initial literature search identified 7,453 studies. We excluded 1,931 duplicates and excluded 5,504 studies by title screening because they did not meet the eligibility criteria. Eighteen randomized controlled trials were selected for full-text review, and 15 papers were discarded; 6 trials compared surgery alone and surgery plus postoperative radiation, 2 compared surgery alone and preoperative longcourse radiation plus surgery, 6 compared surgery alone and preoperative short-course radiation and surgery, and one was long-term results of a previous reported trial. Three randomized trials comparing preoperative CRT with postoperative CRT in resectable stage II-III rectal cancer were finally identified; Park et al. [9] in 2011, Roh et al. [10] in 2009, and Sauer et al. [5] in 2004 (Fig. 1). All patients in both preoperative and postoperative arms received conventionally fractionated radiotherapy of 50-54 Gy with 1.8-2 Gy per fraction for 5-6 weeks. Concurrent chemotherapy of intravenous 5-fluorouracil

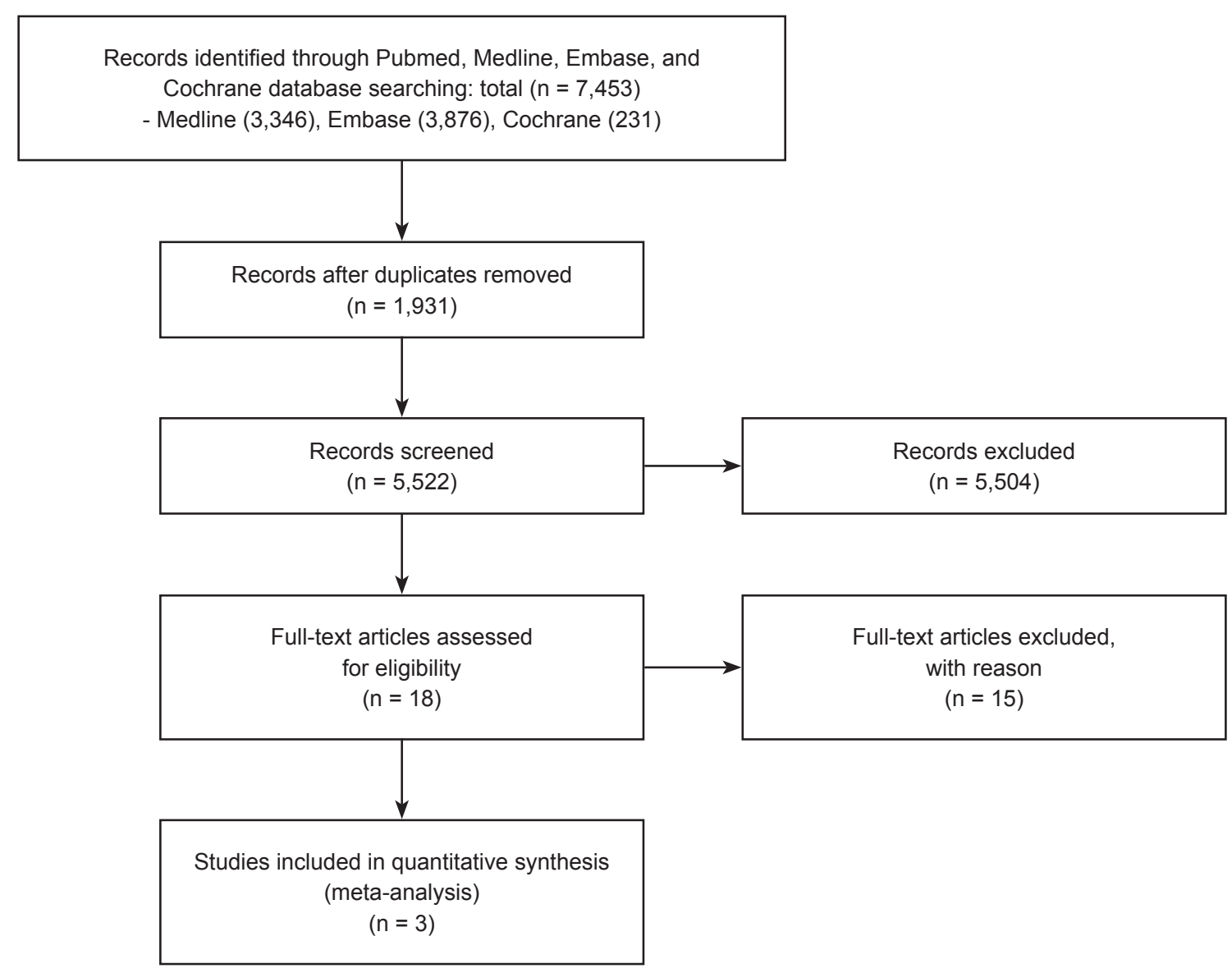

Fig. 1. Flow chart of the search strategy. 


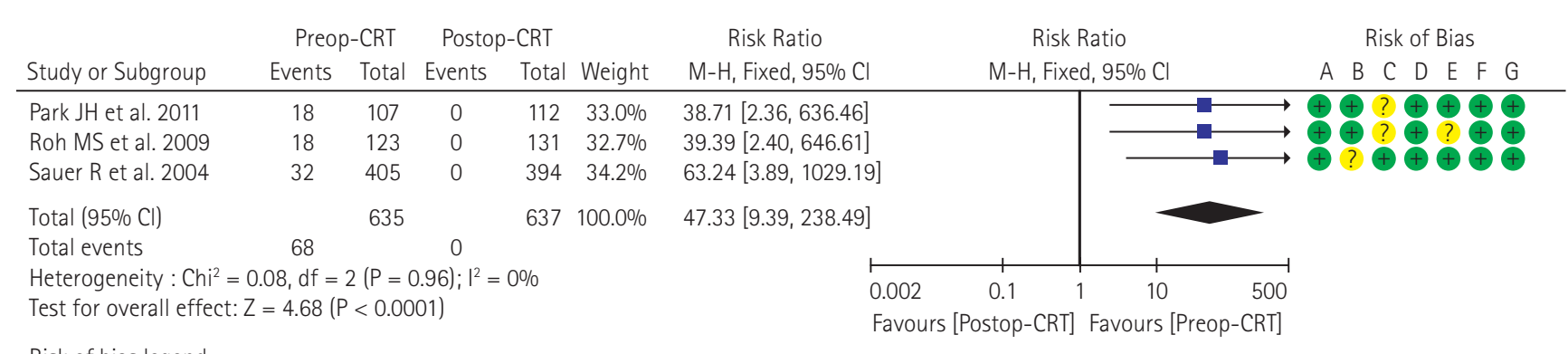

Risk of bias legend

(A) Random sequence generation (selection bias)

(B) Allocation concealment (selection bias)

(C) Blinding of participants and personnel (performance bias)

(D) Blinding of outcome assessment (detection bias)

(E) Incomplete outcome data (attrition bias)

(F) Selective reporting (reporting bias)

(G) Other bias

Fig. 2. Forest plot of comparison: pathologic complete response (ypTONO) between preoperative and postoperative chemoradiotherapy.

(5-FU) or oral capecitabine was administered during the course of radiotherapy. Curative surgery was performed in all patients 4 to 8 weeks after the end of radiotherapy. Median followup time of each study ranged from 4 to 6 years. The baseline characteristics of included studies were summarized in Table 1. Randomization was adequately performed in three studies with no imbalance between treatment arms, and little risk of bias was found in each study. To calculate the overall effect size for reported oncologic outcomes (recurrence and survival), fixedeffect model was applied since there was no heterogeneity among the studies. In contrast, random-effect model was used to calculate the effect size of sphincter preservation and late complication rates since there were heterogeneities among the studies.

\section{Tumor response, recurrence, and survival}

The $\mathrm{pCR}$ in the preoperative-CRT group of three trials were achieved in 18 (16.8\%) of 107 patients (Park et al. [9]), 18 (14.6\%) of 123 patients (Roh et al. [10]), and 32 (7.9\%) of 405 patients (Sauer et al. [5]), respectively. The overall reported pCR rate in the three studies was $10.7 \%$ (Fig. 2).

Table 2 shows reported oncologic results of each study. The locoregional recurrence occurred in 42 (6.6\%) of 635 patients for the preoperative-CRT group. For the postoperative-CRT group, 72 (11.3\%) of 637 patients developed locoregional recurrences. The difference of locoregional recurrence rate at 5 years was statistically significant between preoperative and postoperative-CRT groups ( $\mathrm{HR}, 0.59 ; 95 \% \mathrm{Cl}, 0.41-0.84 ; \mathrm{p}=$ 0.004) (Fig. 3A). However, distant recurrence rate at 5 years was not significantly different between two groups ( $H R$, 0.95;
95\% Cl, 0.80-1.13; $p=0.55$ ) (Fig. 3B). Two trials (Park et al. [9] and Sauer et al. [5]) reporting the 5-year distant recurrence rate were analyzed in this study. There was no significant difference for RFS ( $H R, 0.89 ; 95 \% \mathrm{Cl}, 0.76-1.04 ; p=0.14)$ and $\mathrm{OS}(\mathrm{HR}, 0.89 ; 95 \% \mathrm{Cl}, 0.73-1.08 ; \mathrm{p}=0.22)$ between two groups (Fig. 3C and 3D). Since distant failure, not locoregional failure was the major failure pattern in all three studies, the similar distant failure rate in the included trials resulted in no significant difference in RFS and OS rates between two arms.

\section{Sphincter preservation}

In all three trials, 436 (68.7\%) of 635 patients in the preoperative-CRT group and 412 (64.7\%) of 637 patients in the postoperative-CRT group were able to save their anal sphincters, respectively. The anal sphincter preservation rate in all rectal cancer patients was not significantly different in both groups ( $\mathrm{HR}, 1.13 ; 95 \% \mathrm{Cl}, 0.92-1.40 ; \mathrm{p}=0.24)$ (Fig. 4A). However, the conversion rate from APR to LAR in low-lying rectal tumors (i.e., anal verge $<5 \mathrm{~cm}$ ) was significantly higher in the preoperative-CRT group than in the postoperativeCRT group ( $\mathrm{HR}, 1.78 ; 95 \% \mathrm{Cl}, 1.31-2.41 ; \mathrm{p}<0.001)$ (Fig. 4B). The conversion, in which APR had been anticipated by the preoperative clinical information and LAR that was actually performed, was reported in two studies (Park et al. [9] and Sauer et al. [5]).

\section{Acute and chronic complication}

All three studies reported the incidence of grade 3 or higher acute and long-term toxicities during and after CRT and curative surgery. The acute toxicity in the meta-analysis was 


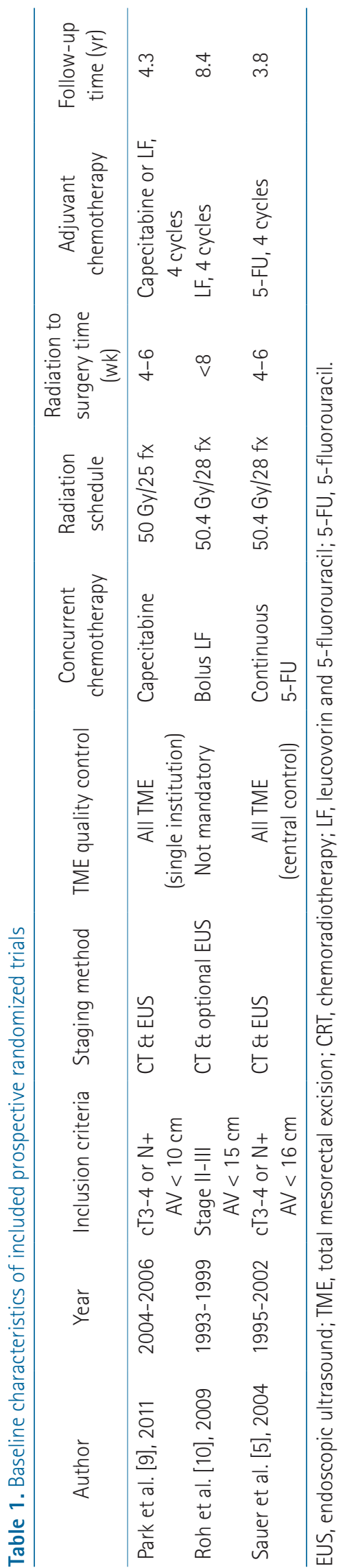

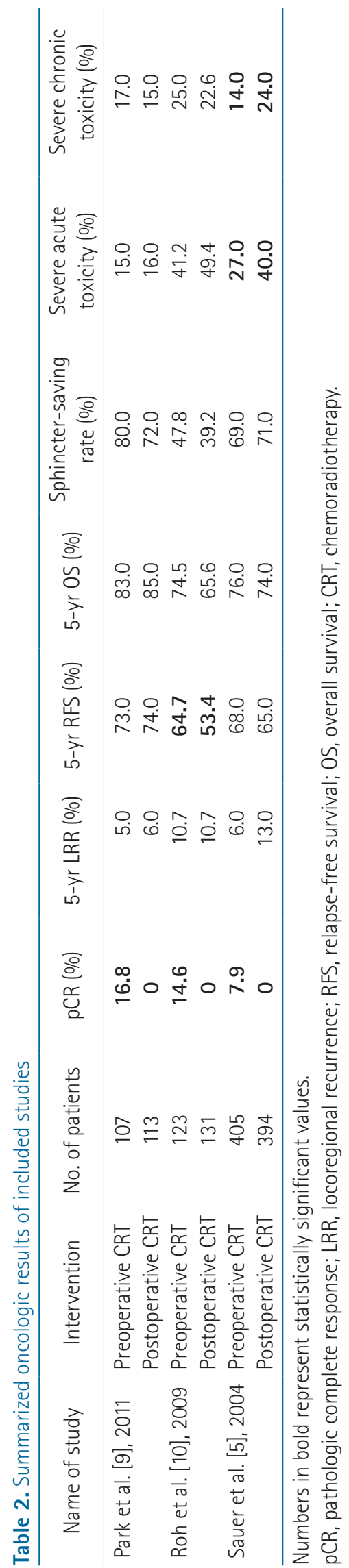

https://doi.org/10.3857/roj.2017.00059 


\section{A. 5-year locoregional recurrence}

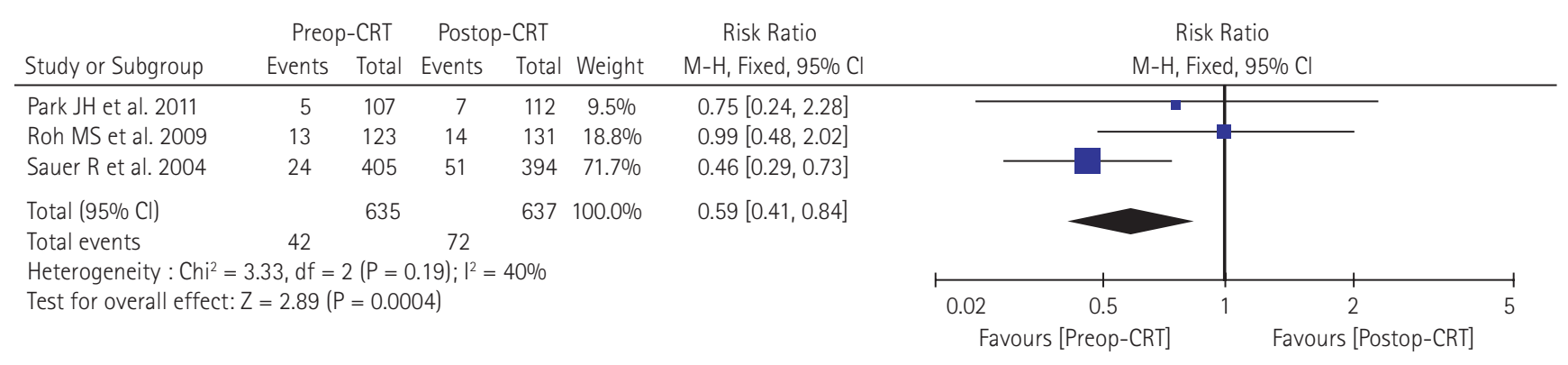

\section{B. 5-year distant recurrence}

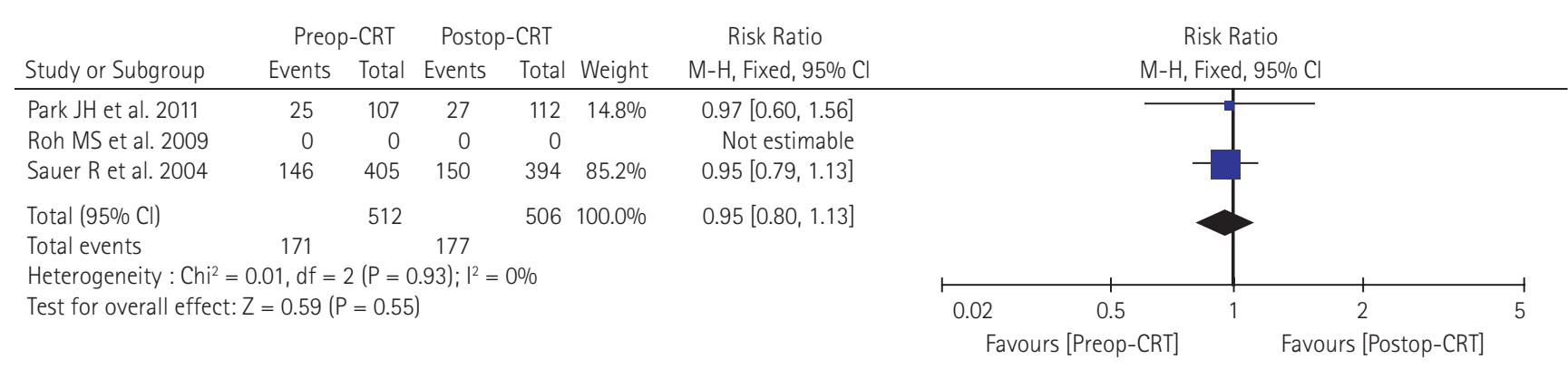

\section{5-year relapse-free survival}

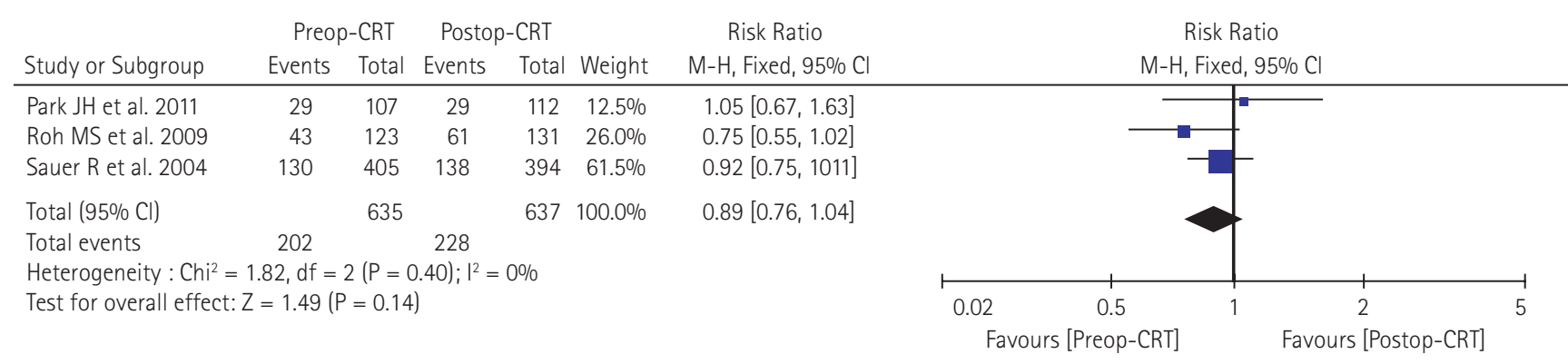

\section{5-year overall survival}

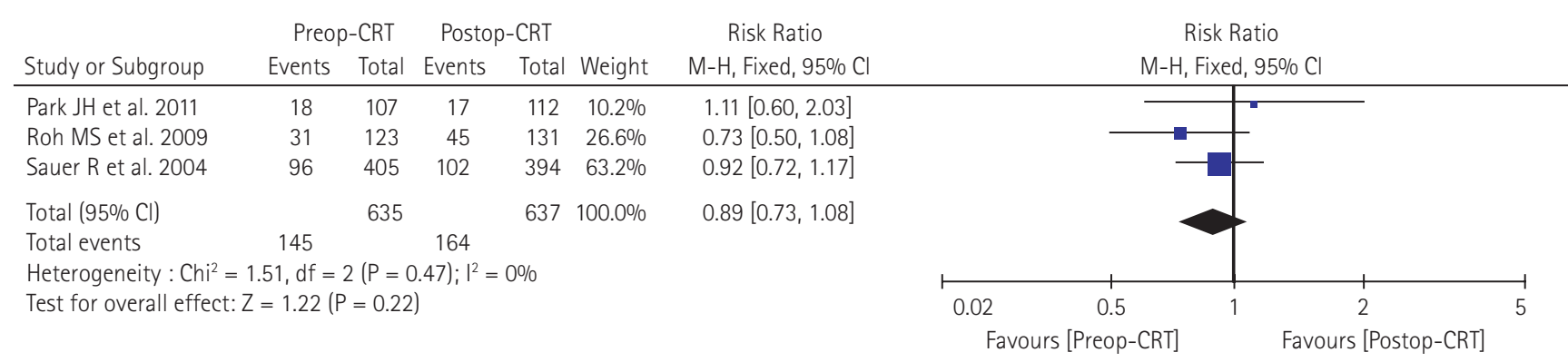

Fig. 3. Forest plot of comparison: (A) locoregional recurrence rate, (B) distant recurrence rate, $(C)$ relapse-free survival, and (D) overall survival between preoperative chemoradiotherapy and postoperative chemoradiotherapy.

significantly lower in the preoperative-CRT group, as compared to the postoperative-CRT group ( $\mathrm{HR}, 0.73 ; 95 \% \mathrm{Cl}, 0.63-0.86$; $p<0.001$ ) (Fig. 5A). However, in terms of perioperative and chronic complication, there was no significant difference between two groups ( $H R, 0.86 ; 95 \% \mathrm{Cl}, 0.53-1.38 ; \mathrm{p}=0.53)$ (Fig. 5B). Sauer et al. [5] showed significantly lower chronic complication rate in the preoperative-CRT group than in the postoperative-CRT group $(p=0.01)$. On the contrary, the 


\section{A. sphincter preservation rate}

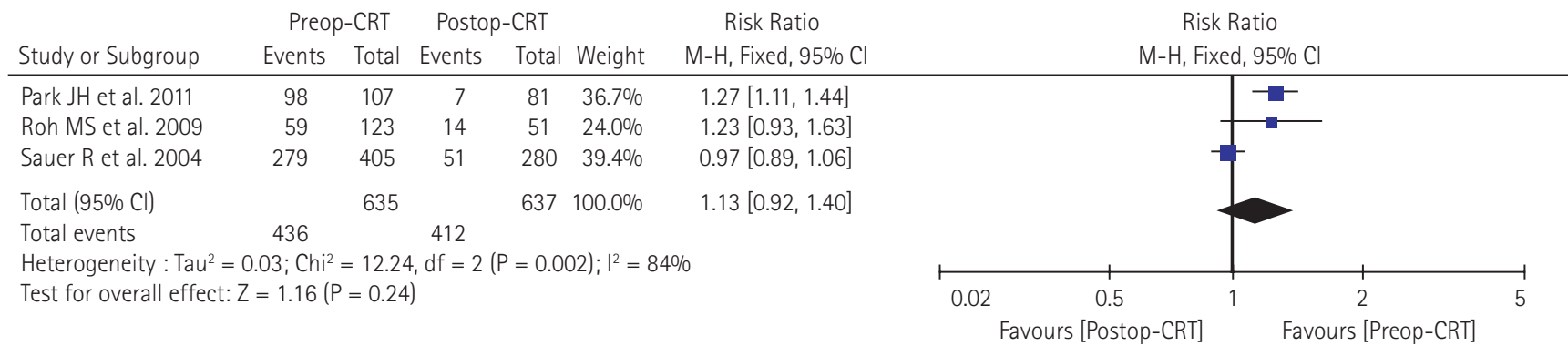

\section{B. conversion rate from APR to LAR}

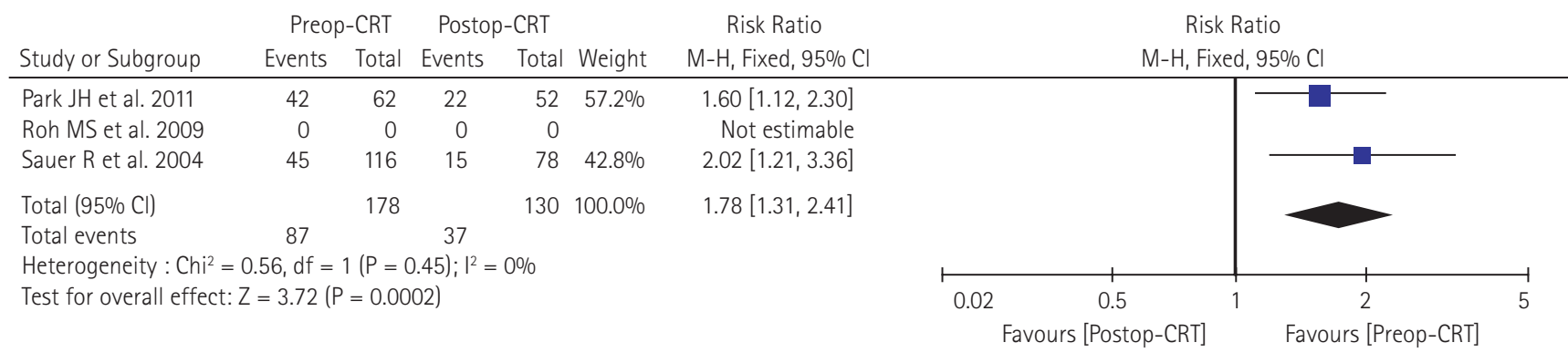

Fig. 4. Forest plot of comparison: (A) anal sphincter preservation rate and (B) surgery conversion rate between preoperative chemoradiotherapy and postoperative chemoradiotherapy.

\section{A. $\geq$ grade 3 acute complication}

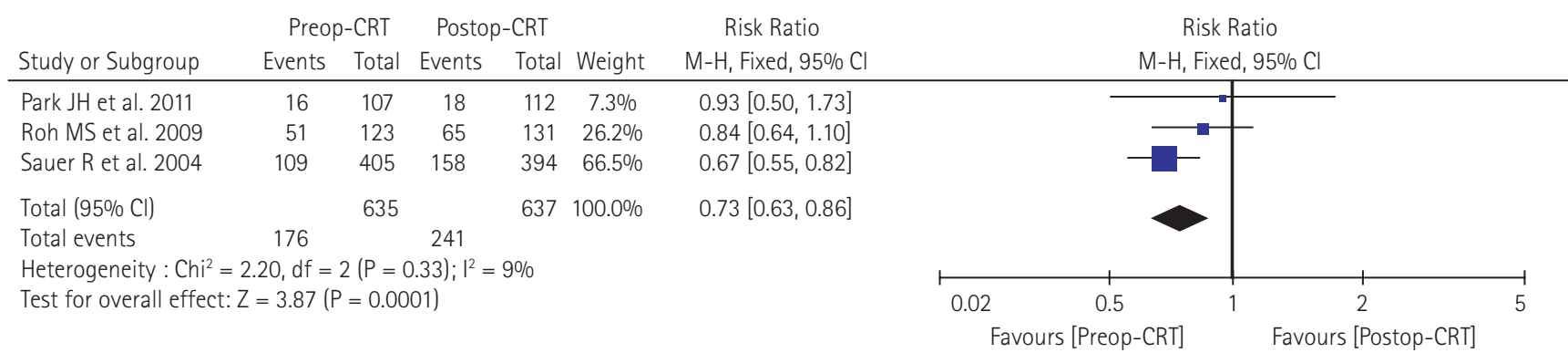

\section{B. $\geq$ grade 3 perioperative or chronic complication}

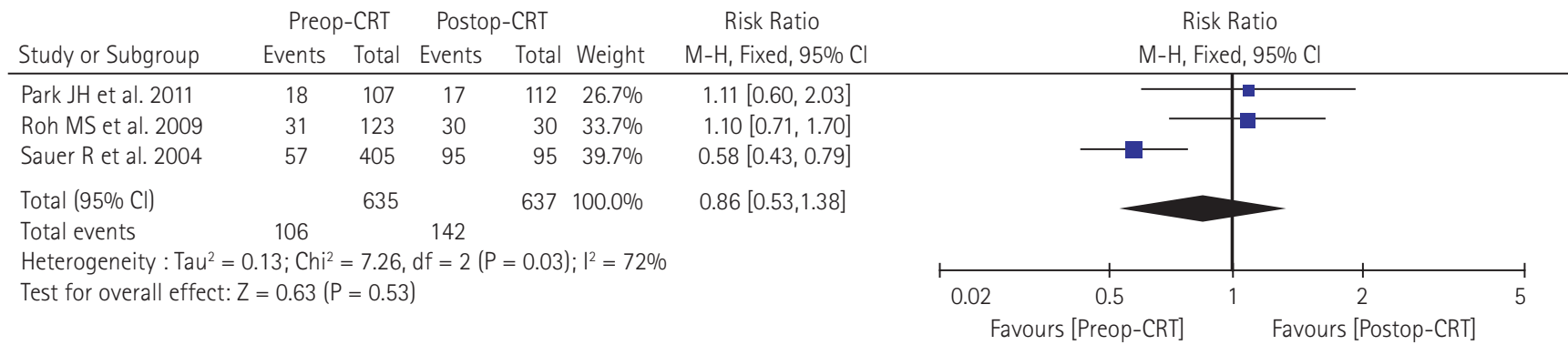

Fig. 5. Forest plot of comparison: (A) grade 3 or higher acute complication and (B) grade 3 or higher perioperative and chronic complication between preoperative chemoradiotherapy and postoperative chemoradiotherapy. 
other two studies showed higher complication rate in the preoperative-CRT group than in the preoperative-CRT group without statistical significance. Due to these mixing results, the meta-analysis results showed no significant difference in terms of perioperative and chronic complication rate between two groups.

\section{Discussion and Conclusion}

For the management of rectal cancer, the most important advancement over the last few decades is the introduction of total mesorectal excision (TME) [11]. TME with sufficient circumferential margin has reduced the local recurrence rate to $<10 \%[2,11]$. There have also been advances in the use of adjuvant radiotherapy and chemotherapy. Several adjuvant randomized studies, such as EORTC 22941 and FFCD 9203, tested different combinations of radiotherapy and chemotherapy. The best results were shown in the concurrent use of radiotherapy and 5-FU-based chemotherapy [12,13].

However, these trials did not conclude whether CRT would undergo before or after curative surgery. The biggest problem to use preoperative CRT is the inaccuracy of the clinical staging $[2,7,8]$. There can be a chance of over-treating an early rectal cancer patient who is not necessary to receive chemoradiotherapy. Actually, in the German trial, 18\% of patients staged clinically as having cT3-4 or node-positive disease were over-staged when their pathologic specimens in the postoperative-CRT group were analyzed $[5,6]$. In addition, some studies suggest that preoperative-CRT is not necessary in patients who have clinical T3N0 stage or have upper rectal cancer $[14,15]$, since, in these patients, the local recurrence rate might be low. Peng et al. [14] showed that 10-year causespecific survival was only improved by postoperative radiation and not by preoperative radiation in clinical T3NO patients based on the Surveillance, Epidemiology, and End Results (SEER) data analysis.

However, there are theoretical and biological potential advantages of the use of preoperative CRT over postoperative CRT $[2,7,8,16,17]$. First, preoperative CRT may sterilize micrometastatic tumor cells earlier than postoperative CRT. Second, usually the radiation field in preoperative CRT is smaller than that of postoperative CRT, and this may reduce toxicity and increase the treatment compliance. Third, better oxygenated tissue of the pelvis before surgery will be significantly more sensitive to an equivalent dose of radiation. Fourth, preoperative CRT will give a better chance of sphincter preservation by tumor downstaging and improving the resectability of low rectal tumors. Fifth, preoperative CRT provides an opportunity to study novel chemotherapeutic agents. The $\mathrm{pCR}$ rate is used to assess the tumor response and patient prognosis.

However, there are not definite and sufficient data that support the advantages of preoperative CRT over postoperative CRT in disease-free survival and overall survival. Only four randomized trials have been performed of this issue. Two trials from United States (Intergroup 0147 and NSABP RO03) were closed early because of limited patient accrual, and only the results of NSABP RO-03 have been reported and included in this meta-analysis [10]. Another is the CAO/ARO/ AIO 93 trial from Germany, which is the landmark study that supports the use of preoperative CRT, and early and longterm results have been reported two times $[5,6]$. The other is the randomized trial from Korea, which was also closed early due to slow enrollments [9]. Thus, only three randomized trials have been evaluated. This meta-analysis demonstrated that there was no significant difference in distant failure rate, RFS, and $\mathrm{OS}$ between preoperative and postoperative-CRT groups. However, the locoregional control and conversion rate of APR to LAR in low rectal tumors were significantly higher in the preoperative-CRT group than in the postoperative-CRT group. In terms of adverse events, the grade 3 or higher acute toxicity was significantly lower in the preoperative-CRT group, while the late toxicity did not show a significant difference between two groups. Although the number of randomized trials addressing the issue of preoperative CRT versus postoperative CRT in rectal cancer is limited, this meta-analysis showed the clinical advantages of preoperative CRT in terms of locoregional control and sphincter preservation with similar chronic toxicities, as compared to postoperative CRT.

The findings of this study must be considered in the context of its limitations. The major limitation of this study is the small number of randomized trials included. Furthermore, the patient number of the German trial was high compared to the others, which resulted in a greater weight being attributed to German trial's conclusions [5]. Other inherent limitations include the risk of heterogeneity among the trials. To reduce the heterogeneity of the trials, we only included three trials which dealt with stage II-III rectal cancer patients with longcourse CRT and curative surgery.

However, the difference of defining the rectum (the distal end from anal verge), staging method, and concurrent chemotherapy regimen could not avoid the inherent heterogeneity. Although preoperative CRT showed non-inferior oncologic outcomes compared to postoperative CRT in all three 
studies, the specific results differ among studies. In contrast to the German study, which showed increased local control rate with no difference in survival rates, the NSABP trial showed better RFS and a trend of improved OS in preoperative-CRT arm than in postoperative-CRT arm. Although the Korean study showed no difference in recurrence or survival, it definitively showed patients treated with preoperative CRT in low-lying tumors ( $<5 \mathrm{~cm}$ from anal verge) had higher rates of sphincter preservation than those treated with postoperative CRT. This result was consistent to that of German study, which also showed higher sphincter preservation rates in low-lying tumors (declared by the surgeon prior to randomization to require an APR). In the NSABP trial, the sphincter preservation rate was higher with no statistical significance in the preoperative-CRT arm (47.8\% vs. $39.2 \% ; p=0.227)$. The difference of treatment compliance, which is the most critical point of the German trial, also differed among the included studies. The compliance rate who completed the CRT was only $54 \%$ in the postoperative-CRT arm in the German study, while $92 \%$ of patients completed the CRT in the preoperativeCRT arm. The compliance rate to the CRT in the Korean study was 76\% in the postoperative-CRT arm and $99 \%$ in the preoperative-CRT arm.

Although some limitations exist in this study, it is to date the only meta-analysis to assess the preoperative and postoperative CRT in the setting with long-course CRT and curative surgery [18-20]. No randomized trial for this issue is in process. Even though the German trial has been criticized based on several issues, NCCN guidelines definitely recommend preoperative CRT as the standard treatment based on the result of the German trial. Thus, we performed this metaanalysis to compare preoperative CRT and postoperative CRT in rectal cancer.

In conclusion, preoperative CRT improves locoregional control, not distant control and survival, with similar chronic toxicity in rectal cancer patients. However, it can give a better chance of sphincter preservation with lower acute toxicity only in low-lying tumors compared to postoperative CRT. Thus, preoperative CRT is recommended as a standard treatment in low rectal cancer.

\section{Conflict of Interest}

No potential conflict of interest relevant to this article was reported.

\section{Acknowledgments}

This work was supported by a Grant from the National RetD Program for Ministry of Science and ICR, and Future Planning, Republic of Korea (No. 2017R1C1B5015762). The statistical analyses performed in this article were advised by Catholic Medical Center Clinical Research Coordinating Center.

\section{References}

1. Siegel R, Desantis C, Jemal A. Colorectal cancer statistics, 2014. CA Cancer I Clin 2014;64:104-17.

2. Willett $C G$, Czito $B G$, Bendell JC. Radiation therapy in stage II and III rectal cancer. Clin Cancer Res 2007;13(22 Pt 2):6903s$8 \mathrm{~s}$.

3. Krook JE, Moertel $C G$, Gunderson $L L$, et al. Effective surgical adjuvant therapy for high-risk rectal carcinoma. N Engl J Med 1991;324:709-15.

4. Wolmark N, Wieand HS, Hyams DM, et al. Randomized trial of postoperative adjuvant chemotherapy with or without radiotherapy for carcinoma of the rectum: National Surgical Adjuvant Breast and Bowel Project Protocol R-02. J Natl Cancer Inst 2000;92:388-96.

5. Sauer $R$, Becker $H_{1}$ Hohenberger W, et al. Preoperative versus postoperative chemoradiotherapy for rectal cancer. N Engl J Med 2004;351:1731-40.

6. Sauer R, Liersch T, Merkel S, et al. Preoperative versus postoperative chemoradiotherapy for locally advanced rectal cancer: results of the German CAO/ARO/AIO-94 randomized phase III trial after a median follow-up of 11 years. J Clin Oncol 2012;30:1926-33.

7. Kachnic LA. Should preoperative or postoperative therapy be administered in the management of rectal cancer? Semin Oncol 2006;33(6 Suppl 11):S64-9.

8. Pasetto LM. Preoperative versus postoperative treatment for locally advanced rectal carcinoma. Future Oncol 2005;1:20920.

9. Park JH, Yoon SM, Yu CS, Kim JH, Kim TW, Kim JC. Randomized phase 3 trial comparing preoperative and postoperative chemoradiotherapy with capecitabine for locally advanced rectal cancer. Cancer 2011;117:3703-12.

10. Roh MS, Colangelo LH, O'Connell MJ, et al. Preoperative multimodality therapy improves disease-free survival in patients with carcinoma of the rectum: NSABP R-03. J Clin Oncol 2009;27:5124-30.

11. van Gijn W, Marijnen CA, Nagtegaal ID, et al. Preoperative radiotherapy combined with total mesorectal excision for 
resectable rectal cancer: 12-year follow-up of the multicentre, randomized controlled TME trial. Lancet Oncol 2011;12:57582.

12. Gerard JP, Conroy T, Bonnetain F, et al. Preoperative radiotherapy with or without concurrent fluorouracil and leucovorin in T3-4 rectal cancers: results of FFCD 9203. J Clin Oncol 2006;24:4620-5.

13. Collette $L$, Bosset JF, den Dulk $M$, et al. Patients with curative resection of cT3-4 rectal cancer after preoperative radiotherapy or radiochemotherapy: does anybody benefit from adjuvant fluorouracil-based chemotherapy? A trial of the European Organisation for Research and Treatment of Cancer Radiation Oncology Group. J Clin Oncol 2007;25:4379-86.

14. Peng LC, Milsom J, Garrett K, et al. Surveillance, epidemiology, and end results-based analysis of the impact of preoperative or postoperative radiotherapy on survival outcomes for T3NO rectal cancer. Cancer Epidemiol 2014;38:73-8.

15. Marinello FG, Frasson M, Baguena G, et al. Selective approach for upper rectal cancer treatment: total mesorectal excision and preoperative chemoradiation are seldom necessary. Dis Colon Rectum 2015;58:556-65.

16. Sebag-Montefiore D, Stephens RJ, Steele R, et al. Preoperative radiotherapy versus selective postoperative chemoradiotherapy in patients with rectal cancer (MRC CRO7 and NCIC-CTG C016): a multicentre, randomised trial. Lancet 2009;373:811-20.

17. Lee JW, Lee JH, Kim JG, et al. Comparison between preoperative and postoperative concurrent chemoradiotherapy for rectal cancer: an institutional analysis. Radiat Oncol J 2013;31:15561.

18. Lee JH, Kim DY, Kim SH, et al. Carcinoembryonic antigen has prognostic value for tumor downstaging and recurrence in rectal cancer after preoperative chemoradiotherapy and curative surgery: a multi-institutional and case-matched control study of KROG 14-12. Radiother Oncol 2015;116:2028.

19. Song JH, Kim SH, Lee JH, et al. Significance of histologic tumor grade in rectal cancer treated with preoperative chemoradiotherapy followed by curative surgery: a multi-institutional retrospective study. Radiother Oncol 2016;118:387-92.

20. Kwak YK, Kim K, Lee JH, et al. Timely tumor response analysis after preoperative chemoradiotherapy and curative surgery in locally advanced rectal cancer: a multi-institutional study for optimal surgical timing in rectal cancer. Radiother Oncol 2016;119:512-8. 